\title{
Intersection topologies with respect to separable GO-spaces and the countable ordinals
}

\author{
by
}

\author{
M. R. J ones (Oxford)
}

\begin{abstract}
Given two topologies, $\mathcal{T}_{1}$ and $\mathcal{T}_{2}$, on the same set $X$, the intersection topology with respect to $\mathcal{T}_{1}$ and $\mathcal{T}_{2}$ is the topology with basis $\left\{U_{1} \cap U_{2}: U_{1} \in \mathcal{T}_{1}, U_{2} \in \mathcal{T}_{2}\right\}$. Equivalently, $\mathcal{T}$ is the join of $\mathcal{T}_{1}$ and $\mathcal{T}_{2}$ in the lattice of topologies on the set $X$.

Following the work of Reed concerning intersection topologies with respect to the real line and the countable ordinals, Kunen made an extensive investigation of normality, perfectness and $\omega_{1}$-compactness in this class of topologies. We demonstrate that the majority of his results generalise to the intersection topology with respect to an arbitrary separable GO-space and $\omega_{1}$, employing a well-behaved second countable subtopology of the separable GO-space.
\end{abstract}

1. Introduction. This paper considers the results of Kunen [3] regarding intersection topologies with respect to the real line and the countable ordinals, and demonstrates how they may be generalised to the intersection topology with respect to an arbitrary separable GO-space and $\omega_{1}$. In so doing, it also generalises the results of the author regarding the intersection topology with respect to the Sorgenfrey line and $\omega_{1}[1]$.

The study of intersection topologies was initiated by G. M. Reed in [5]. He makes an extensive study of the class $\mathcal{C}$ of intersection topologies with respect to the real-line topology and an $\omega_{1}$-type order topology on subsets of $\mathbb{R}$ of cardinality $\aleph_{1}$. Kunen studied the class further, establishing that the properties of normality, "perfectness" and $\omega_{1}$-compactness in $\mathcal{C}$ are closely inter-related and dependent on the model of set theory used.

In [1], we succeeded in showing that many of the properties of $\mathcal{C}$ also hold in the class $\mathcal{S}$ of intersection topologies with respect to the Sorgenfrey line topology and an $\omega_{1}$-type order topology. Although Reed's results depended primarily on the hereditary separability of the ordinary Euclidean topology,

1991 Mathematics Subject Classification: Primary 54A10; Secondary 54D15, 54F05.

Key words and phrases: intersection topology, GO-space, separable, subtopology, normality, $\omega_{1}$-compactness, countable ordinals. 
and thus carried over to the Sorgenfrey case relatively easily, Kunen's results were more difficult to generalise, and relied heavily on particular properties of the Sorgenfrey line. First, we use the submetrizability of the Sorgenfrey line, and, moreover, particularly "close" submetrizability, in the sense that basic open sets in the Sorgenfrey line and those in the real line differ by only one point (the "end-point" of the half-open Sorgenfrey interval). Second is the orderability of the Sorgenfrey line. And lastly, we use its separability.

Generalising these results to the intersection topology of an arbitrary separable GO-space and $\omega_{1}$ seemed reasonable, but the problem that immediately becomes apparent is that in the general case there is no obvious metrizable topology to employ. Indeed, separable GO-spaces are not in general submetrizable: the Alexandrov double arrow space (the space $[0,1] \times\{0,1\}$ equipped with the lexicographic order topology) is a compact and separable LOTS but does not have a countable base, so is not submetrizable.

However, there are some well-behaved topologies on the set $[0,1] \times\{0,1\}$. First is the ordinary Euclidean topology (inherited by $[0,1] \times\{0,1\}$ as a subspace of $\mathbb{R}^{2}$ ), but the problem here is that the topology is too coarse an approximation-Euclidean-open sets like $[0,1] \times\{0\}$ have empty interior w.r.t. the double arrow topology - and it even fails to be a subtopology of the double arrow space. To remedy this, we consider instead the rather odd (not even $T_{0}$ !) subtopology

$$
\mathcal{T}=\left\{U \times\{0,1\}: U \in \mathcal{T}_{\mathbb{R}}\right\}
$$

where $\mathcal{T}_{\mathbb{R}}$ is the Euclidean topology on $[0,1]$. Evidently this topology is "close" to the double arrow topology in the same way that the Sorgenfrey line is "close" to the real line. And although it fails to be metrizable, it is second countable, and this turns out to be enough.

It is surprising to discover that a similar process can be applied to an arbitrary separable GO-space. In this paper, we show that any separable GO-space, $(X, \mathcal{T})$, has a second countable subtopology $\mathcal{M}$ with the property that if $\mathcal{T}_{\omega_{1}}$ is an $\omega_{1}$-type order topology on $X$, and if $U$ is a $\mathcal{T} \vee \mathcal{T}_{\omega_{1}}$-open set, then there is an $\mathcal{M}$-open $V$ such that $U \backslash V$ is nonstationary and $V \backslash U$ is countable. This result allows us to carry Kunen's results over into this more general setting.

Preliminaries. An $\omega_{1}$-type order topology on a set $X$ will always be denoted by $\mathcal{T}_{\omega_{1}}$. If we refer to $\left(X, \mathcal{T}_{\omega_{1}}\right)$ then implicitly it will be assumed that $X$ has been equipped with some $\omega_{1}$-type order as

$$
X=\left\{x_{\alpha}: \alpha<\omega_{1}\right\} .
$$

So we will call a subset of $X$ stationary or $c l u b$ if it has the corresponding property with respect to the $\omega_{1}$-type order, and sometimes we use the short- 
hand $(\alpha, \beta]$ to denote the set $\left\{x_{\theta}: \alpha<\theta \leq \beta\right\}$, and so on. For $x, y \in X$ we say $x<y$ in $\omega_{1}$ if $\beta<\alpha$, where $x=x_{\beta}$ and $y=x_{\alpha}$. We shall occasionally use the convention that, for $x \in X, \alpha_{x} \in \omega_{1}$ is such that $x=x_{\alpha_{x}}$.

Recall the definition of the intersection topology:

Definition 1.1. If $\mathcal{T}_{1}$ and $\mathcal{T}_{2}$ are topologies defined on the set $X$, then the intersection topology, denoted by $\mathcal{T}_{1} \vee \mathcal{T}_{2}$, with respect to $\mathcal{T}_{1}$ and $\mathcal{T}_{2}$ is the topology on $X$ with basis

$$
\left\{U_{1} \cap U_{2}: U_{1} \in \mathcal{T}_{1}, U_{2} \in \mathcal{T}_{2}\right\} .
$$

2. The subtopology $\mathcal{M}$. The following fact can be derived from a result of Ostaszewski [4]. We give a direct proof, and then use this property to construct the subtopology $\mathcal{M}$.

Lemma 2.1. Let $(X, \mathcal{T})$ be a separable GO-space with underlying order $\leq$. Then there exists a nondecreasing continuous function $\phi: X \rightarrow[0,1]$ such that $\phi(x)=\phi(y)$ implies $(x, y)=\emptyset$.

Proof. Let $a<b$. A standard proof of Urysohn's Lemma (e.g. [2, Chap. 4, Lemma 4]) gives a nondecreasing order-continuous function $\lambda$ : $X \rightarrow[0,1]$ such that $\lambda(a)=0$ and $\lambda(b)=1$. (Indeed, if there are $u<v$ with $a \leq u<v \leq b$ and $(u, v)=\emptyset$, let $\lambda$ be 0 on $(\leftarrow, u]$ and 1 on $[v, \rightarrow)$. If this is not the case, choose inductively, for dyadic rationals $r=p 2^{-q} \in(0,1)$, elements $u_{r} \in(a, b)$ with $u_{r}<u_{s}$ whenever $r<s$, and set $\lambda(x)=\inf \{r$ : $\left.x<u_{r}\right\}$ if such $u_{r}$ exist, or $\lambda(x)=1$ otherwise.)

Now, let $D$ be a countable set dense in $X$. For each pair $a<b$ from $D$, fix a function $\lambda$ as above, and for each $a \in D$ isolated in $(X, \mathcal{T})$, let $\lambda$ be the characteristic function of $[a, \rightarrow)$. Then arrange the functions $\lambda$ into a sequence $\lambda_{1}, \lambda_{2}, \ldots$ and set

$$
\phi=\sum_{i} 2^{-i} \lambda_{i}
$$

We can now define our topology $\mathcal{M}$ :

Lemma 2.2. Let $\mathcal{M}$ be the topology on $X$ defined by $\mathcal{M}=\left\{\phi^{-1}(G)\right.$ : $G$ is open in $[0,1]\}$. Then $(X, \mathcal{M})$ is a second countable subtopology of $\mathcal{T}$.

The reason for constructing this subtopology will become clear in the next section, where we derive results about the intersection topology with $\omega_{1}$.

3. Applications to the intersection topology. Having constructed the subtopology $\mathcal{M}$, we can now use it to derive results about the intersection topology with $\omega_{1}$.

Lemma 3.1 If $U$ is $\mathcal{T} \vee \mathcal{T}_{\omega_{1}}$-open, then there is an $\mathcal{M}$-open $V$ such that $U \backslash V$ is nonstationary and $V \backslash U$ is countable. 
If $H$ is $\mathcal{T} \vee \mathcal{T}_{\omega_{1}}$-closed, then there is an $\mathcal{M}$-closed $K$ such that $K \backslash H$ is nonstationary and $H \backslash K$ is countable.

P r o of. Obviously, the second statement follows from the first.

Let $\phi: X \rightarrow[0,1]$ be as in Lemma 2.1 , and set

$$
M=\bigcup\left\{(r, s): \phi^{-1}(r, s) \backslash U \text { is countable with } r<s \text { rational }\right\} .
$$

Note that $M$ is open in $[0,1]$ and that $\phi^{-1}(M) \backslash U$ is countable. Next we show that $U \backslash \phi^{-1}(M)$ is nonstationary.

Let $W=U \backslash \phi^{-1}(M)$, and suppose, for a contradicton, that $W$ is stationary. For each non-isolated $x_{\xi} \in W$ choose $f(\xi)<\xi$ and an interval $\left[a_{\xi}, b_{\xi}\right]$ such that $x_{\xi} \in\left\{a_{\xi}, b_{\xi}\right\},\left(a_{\xi}, b_{\xi}\right) \neq \emptyset$ and $\left[a_{\xi}, b_{\xi}\right] \cap(f(\xi), \xi] \subseteq U$. Without loss of generality, using the Pressing Down Lemma, one can assume that for an uncountable set $A \subseteq W$ and $\gamma<\omega_{1}$ we have $f(\xi)=\gamma$ and $x_{\xi}=b_{\xi}$ for $x_{\xi} \in A$, i.e.

$$
\left[a_{\xi}, x_{\xi}\right] \cap(\gamma, \xi] \subseteq U \quad \text { and } \quad \phi\left(a_{\xi}\right)<\phi\left(x_{\xi}\right) \quad \text { for } x_{\xi} \in A .
$$

There exist $x_{\alpha} \in A$, uncountable $C \subseteq A$, and rationals $r, s$ such that

$$
\phi\left(a_{\xi}\right)<r<\phi\left(x_{\alpha}\right)<s<\phi\left(x_{\xi}\right) \quad \text { for } x_{\xi} \in C \text {. }
$$

To show this, first choose $\varepsilon>0$ and an uncountable $B \subseteq A$ with $\phi\left(x_{\xi}\right)-$ $\phi\left(a_{\xi}\right)>\varepsilon$ for $x_{\xi} \in B$. Next, use the hereditary Lindelöf property of the Sorgenfrey line topology on $[0,1]$ to find $x_{\alpha} \in B$ and a rational $s \in\left(\phi\left(x_{\alpha}\right), \phi\left(x_{\alpha}\right)\right.$ $+\varepsilon / 2)$ such that the set

$$
C=\left\{x_{\xi} \in B: s<\phi\left(x_{\xi}\right)<\phi\left(x_{\alpha}\right)+\varepsilon / 2\right\}
$$

is uncountable. Finally, take any rational $r \in\left(\phi\left(x_{\alpha}\right)-\varepsilon / 2, \phi\left(x_{\alpha}\right)\right)$.

We claim that

$$
\phi^{-1}(r, s) \backslash U \subseteq(0, \gamma] .
$$

Indeed, if $\phi\left(x_{\beta}\right) \in(r, s)$ and $\beta>\gamma$, choose $x_{\xi} \in C$ with $\xi>\beta$ and use (2) and (3) to get $x_{\beta} \in\left[a_{\xi}, x_{\xi}\right] \cap(\gamma, \xi] \subseteq U$. From (4) and (1), $(r, s) \subseteq M$ and therefore, by (3), $x_{\alpha} \in \phi^{-1}(M) \cap W$. But this contradicts the definition of $W$.

Finally, put $V=\phi^{-1}(M)$. Then $V \in \mathcal{M}$ and has the properties required.

This key lemma allows us to generalise all of the results of Kunen's paper [3] to an arbitrary separable GO-space with various modifications of his proofs. Here we give a couple of examples of how the proofs generalise, and quote the remaining results.

Lemma 3.2. If $\left(X, \mathcal{T} \vee \mathcal{T}_{\omega_{1}}\right)$ is $\omega_{1}$-compact, then all nonstationary $\mathcal{M}$ closed sets are countable. 
Proof. Suppose there exists an uncountable nonstationary $\mathcal{M}$-closed set $H$. Then there exists a club set $C$ disjoint from $H$. But then we can let $K$ be an uncountable subset of $H$ such that any two distinct elements of $K$ have an element of $C$ between them. But then $K$ is closed discrete with respect to $\mathcal{M}$, and thus also with respect to $\mathcal{T} \vee \mathcal{T}_{\omega_{1}}$, as $\mathcal{M} \subseteq \mathcal{T} \subseteq \mathcal{T}_{\omega_{1}}$, contradicting $\omega_{1}$-compactness.

The next theorem explores the link between $\omega_{1}$-compactness and normality.

THEOREM 3.3. If $\left(X, \mathcal{T} \vee \mathcal{T}_{\omega_{1}}\right)$ is $\omega_{1}$-compact, then $\left(X, \mathcal{T} \vee \mathcal{T}_{\omega_{1}}\right)$ is normal.

Proof. Let $H_{1}$ and $H_{2}$ be closed and disjoint. By Lemma 3.1, let $K_{1}$ and $K_{2}$ be $\mathcal{M}$-closed with $K_{1} \backslash H_{1}$ and $K_{2} \backslash H_{2}$ nonstationary, $H_{1} \backslash K_{1}$ and $H_{2} \backslash K_{2}$ countable. Then $K_{1} \cap K_{2}$ is nonstationary and hence countable by Lemma 3.2.

Let $\alpha<\omega_{1}$ be such that all elements of $H_{1} \backslash K_{1}, H_{2} \backslash K_{2}$ and $K_{1} \cap K_{2}$ are below $\alpha$. Then $X$ is partitioned into $\mathcal{T}_{\omega_{1}}$-clopen sets $[0, \alpha]$ and $\left[\alpha+1, \omega_{1}\right)$. Provided we can separate $H_{1}$ and $H_{2}$ on each of these, we are done.

- On $[0, \alpha], H_{1}$ and $H_{2}$ can be separated because countable regular spaces are normal.

- On $\left[\alpha+1, \omega_{1}\right), H_{1}$ and $H_{2}$ can be separated because they are contained in disjoint $\mathcal{M}$-closed sets $K_{1}$ and $K_{2}$, which can be separated in $\mathcal{T}$ because $\mathcal{M} \subseteq \mathcal{T}$ and $\mathcal{T}$, being a GO-space topology, is hereditarily normal.

We quote the remaining theorems we have managed to establish. The converse to Theorem 3.3 is not true, but what can be said is

Theorem 3.4. If $\left(X, \mathcal{T} \vee \mathcal{T}_{\omega_{1}}\right)$ is normal, then there is a club $C$ which is $\omega_{1}$-compact in its relative topology.

THEOREM 3.5. (CH) There exists a closed unbounded subset $D$ of $(X$, $\left.\mathcal{T} \vee \mathcal{T}_{\omega_{1}}\right)$ such that $D$ is normal.

TheOREM 3.6. $\left(X, \mathcal{T} \vee \mathcal{T}_{\omega_{1}}\right)$ is not perfectly normal.

TheOREM 3.7. The following are equivalent for $\left(X, \mathcal{T} \vee \mathcal{T}_{\omega_{1}}\right)$ :

1. $\left(X, \mathcal{T} \vee \mathcal{T}_{\omega_{1}}\right)$ is perfect.

2. Every club set is a $G_{\delta}$-set with respect to $\mathcal{T} \vee \mathcal{T}_{\omega_{1}}$.

3. Every club set contains a club set which is a $G_{\delta}$-set with respect to $\mathcal{M}$.

Theorem 3.8. $(\mathbf{C H})\left(X, \mathcal{T} \vee \mathcal{T}_{\omega_{1}}\right)$ is not perfect.

Reed [5] has a theorem asserting that, under $\mathbf{M A}+\neg \mathbf{C H}$, every real$\omega_{1}$ intersection topology is perfect and thus (by Theorem 3.6) not normal. There are examples in the general case we consider here, though, where this is evidently not true. Consider the double arrow space, with underlying set 
$[0,1] \times\{0,1\}$. The analysis of this space in Section 2 may be carried out in such a way as to ensure that the topology $\mathcal{M}$ consists of all sets of the form $U \times\{0,1\}$, where $U$ is open in the Euclidean topology on $[0,1]$. But then $G_{\delta}$-sets with respect to $\mathcal{M}$ are all of the form $Y \times\{0,1\}$ for some $Y \subseteq[0,1]$. Glancing now at Theorem 3.7, consider the possibility that the "top arrow" contains a club set. Then, certainly, this will be a club set which fails to contain a club $G_{\delta}$-set with respect to $\mathcal{M}$, and by Theorem 3.7 therefore fails to be perfect. This, then, is the only situation in which the results of [3] have failed to generalise.

If these results can be generalised to some larger class of spaces, then it is unclear to the author how to do so. Kunen's proofs hold in the general separable metric case, the proofs above hold for all separable GO-spaces, and we have shown that a result analogous to Lemma 3.1 exists for the bow-tie space. But the proofs of this result and of Lemma 3.1 both rely on some kind of order structure in the space, either directly, as in the case of all separable GO-spaces, or indirectly, as with the bow-tie space, where the real line is embedded. Finding some kind of comparable order structure in, say, an arbitrary separable stratifiable or even an arbitrary separable monotonically normal space would appear to be a difficult problem.

\section{References}

[1] M. R. Jones, Sorgenfrey- $\omega_{1}$ intersection topologies, preprint, 1993.

[2] J. L. Kelley, General Topology, Springer, New York, 1975.

[3] K. Kunen, On ordinal-metric intersection topologies, Topology Appl. 22 (1986), $315-319$.

[4] A. J. Ostaszewski, A characterisation of compact, separable, ordered spaces, J. London Math. Soc. 7 (1974), 758-760.

[5] G. M. Reed, The intersection topology with respect to the real line and the countable ordinals, Trans. Amer. Math. Soc. 297 (1986), 509-520.

ST. CROSS COLLEGE

UNIVERSITY OF OXFORD

OXFORD OX1 3LZ

U.K.

Received 13 September 1993;

in revised form 27 April 1994 